\title{
Biliary atresia: Color doppler US findings in neonates and infants at a specialized hospital.
}

\author{
Monowara $\mathbf{M}^{1^{*}}$, Hossain MM${ }^{1}$, Sheuly $\mathbf{A H}^{2}$, Parvej $\mathbf{M}^{3}$, Ipsita Biswas $\mathbf{I}^{4}$ \\ ${ }^{1}$ Department of Radiology and Imaging, Bangladesh Institute of Child Health, Shishu Hospital, Dhaka, Bangladesh \\ ${ }^{2}$ National Institute of Ophthalmology and Hospital, Dhaka, Bangladesh \\ ${ }^{3}$ Department of Pathology, Bangladesh Institute of Child Health, Shishu Hospital, Dhaka, Bangladesh \\ ${ }^{4}$ Department of Pediatric Surgery, Bangladesh Institute of Child Health, Shishu Hospital, Dhaka, Bangladesh
}

\begin{abstract}
Neonatal jaundice occurred due to various causes. Most cholestatic conditions can be classified into either as obstructive or as hepatocellular in origin. More than $90 \%$ of obstructive cholestasis are due to Biliary Atresia (BA). Due to impairment of bile formation usually hepatocellular cholestasis occurs and it indicates that hepatocytes are not functioning properly. The aim of this study was to describe the color Doppler US findings in the livers of neonates in BA with non-BA and control groups.
\end{abstract}

Keywords: Biliary atresia, Neonates, Jaundice, Hepatic arteriopathy, Vein bifurcation.

Accepted on December 05, 2018

\section{Introduction}

Idiopathic neonatal hepatitis causes majority of the hepatocellular cholestasis. For proper management protocol it is important to evaluate either obstructive or hepatocellular cholestasis. Because treatment options for one is surgical and for other is medication $[1,2]$. But from clinical symptoms, biochemical and histological findings, it is very difficult to differentiate between both, as they share almost same features. Therefore, Mayor et al. [3] introduced general guidelines to evaluate cholestatic jaundice in infants and neonates.

In this type of patients, high-spatial-resolution real-time ultrasonography (US) serves as a first-time investigation tool to determine the cause of jaundice. For diagnosis of BA, the presence of Triangular Cord (TC) sign and an abnormal gallbladder (GB) on US image is widely accepted [4]. However, sometimes TC sign may be missed. Its detection is largely dependent on the techniques used by the sinologist, machine and his or her experience. Furthermore, in neonates with congenital anomaly, it is very difficult to find TC sign or if the resolution of the US apparatus is poor [5].

At US, compare to non-BA and control subjects usually, hepatic artery diameter is larger in BA patients. The range of the diameters of the normal hepatic artery is $1.0 \mathrm{~mm}-2.5 \mathrm{~mm}$ according to Kim et al. [6] and $0.5 \mathrm{~mm}-4.0 \mathrm{~mm}$ according to Humphrey and Stringer [7].

\section{Methods}

It was a prospective observational study of 3 years extending from January 2013 to January 2017 at radiology and imaging Department of Dhaka Shishu Hospital. Before staring the study institutional review board approval was obtained and acquisition of informed consent was exempted. In this study US and color Doppler US was done in 35 patients with neonatal cholestasis who had not been fed for at least 4 hours and 30 control neonates who did not have jaundice or liver diseases. The clinical diagnoses of this control group were as follows: gastrointestinal tract disease in thirteen patients, renal disease in ten patients and congenital heart disease in seven patients.

Routine chemical investigations, including measurement of total bilirubin were done for every patient. The reference range for blood sample parameter provided by our institute was 0.2 $1.2 \mathrm{mg} / \mathrm{dl}$ for total bilirubin. One radiologist more than 3 years of experience performed all the US by using 5-8 $\mathrm{MHz}$ curved linear and 5-12 MHz linear transducers (Simenssonoline G-40 and Siemens acuson NX 3 Elite) in all patients.

On US and Color Doppler US, TC sign present or not, GB length in $\mathrm{cm}$, the thickness of the echogenic anterior wall of right portal vein (EARPV) in $\mathrm{mm}^{2}$ just proximal to the right portal vein bifurcation, Diameter of hepatic artery in $\mathrm{mm}^{2}$ and presence or absence of hepatic subscapular flow were recorded (Figure 1). A GB length of $1.5 \mathrm{~cm}$ was considered as normal. In US EARPV thicker than $4 \mathrm{~mm}$ on longitudinal US image was taken as presence of TC sign. The observer measured all the parameters twice and chooses the largest ones. After completion of US all patients were underwent color Doppler US (pulse repetition frequency, $1200-1500 \mathrm{~Hz}$; power gain percentage, 82\%-92\%: Medium flow velocity; medium wall filter). For transverse scanning examination with the linear transducer, the color box was placed on the anterior surface around the falciform ligament. The color box measured $1 \mathrm{~cm}$ in height and $3-4 \mathrm{~cm}$ in width. We considered hepatic subscapular flow to be present when vascular structures continued to the liver sub capsular surface on color Doppler US images (Figure 2). All patient's diagnosis was confirmed by histopathological study.

The two sample $t$ test was done to compare the total bilirubin between BA and those of Non-BA and for age distribution between BA and control groups. For categorical association person $\mathrm{x}^{2}$ test were done. Multivariate logistic regression analysis was done to determine the significant association of 
Citation: Monowara M, Hossain MM, Sheuly AH, et al. Biliary atresia: Color doppler US findings in neonates and infants at a specialized hospital. J Child Adolesc Health. 2018;2(2):27-30.
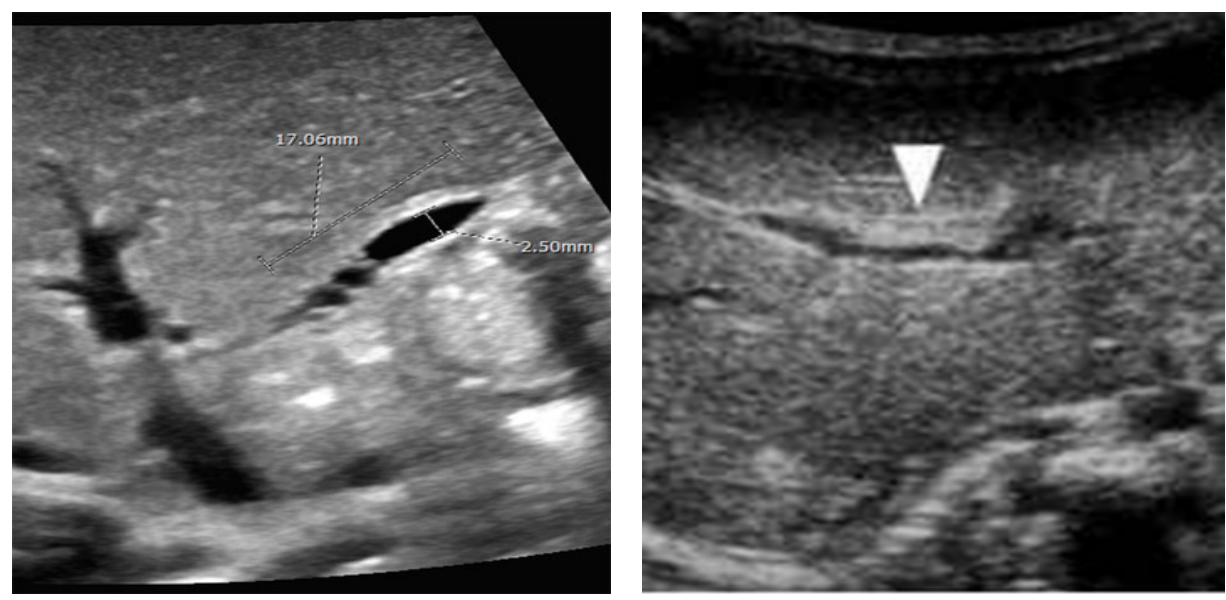

Figure 1. Gall bladder measurement.
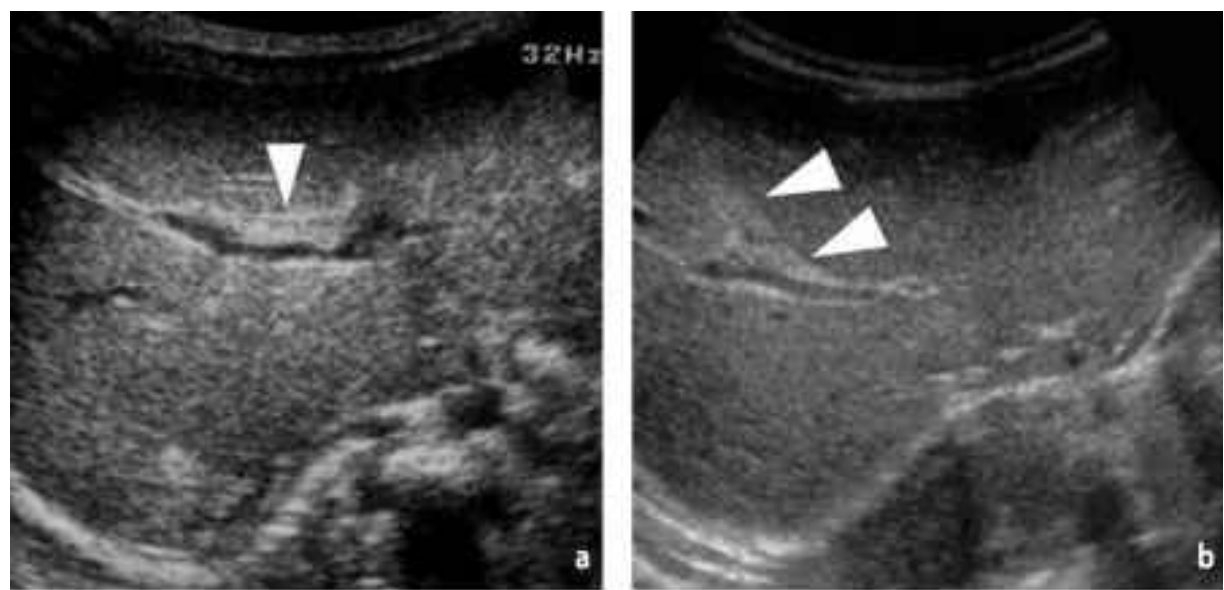

Figure 2. TC sign on US

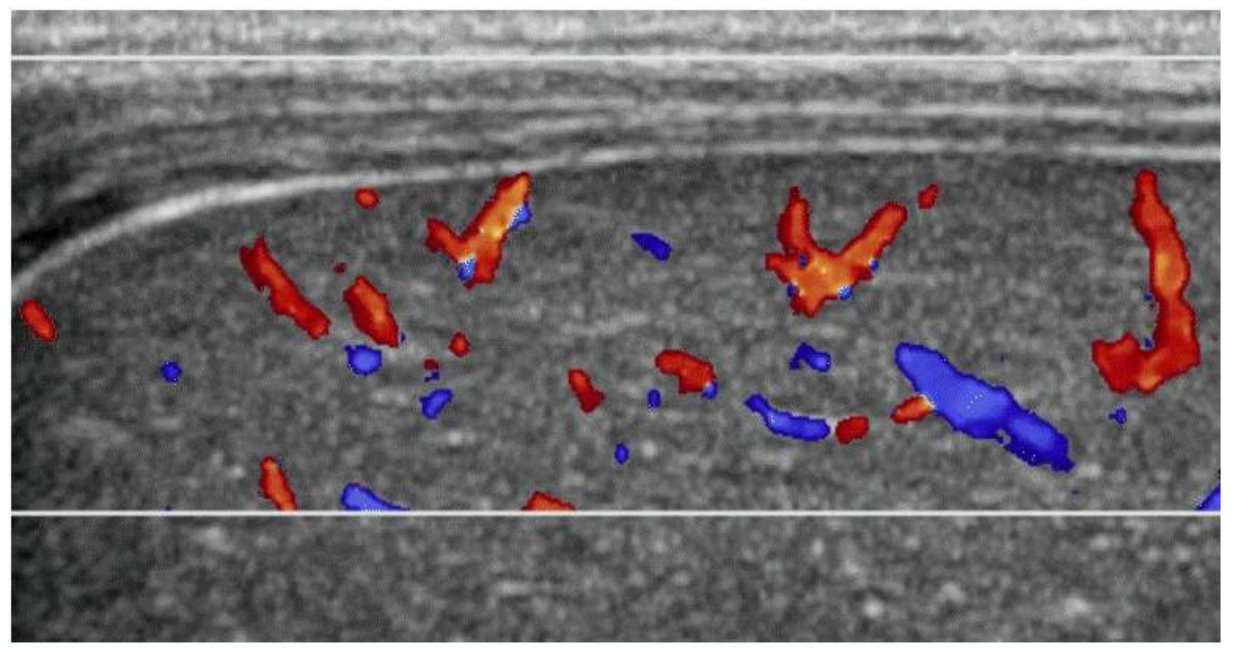

Figure 3. Subscapular flow on colour doppler.

presence of TC sign, GB length, diameter of portal vein and hepatic artery and presence of hepatic subscapular flow were useful with presence or absence of BA. Data analyses were done with statistical software.

\section{Results}

In the current study among the 40 cases after confirmed diagnosis 29 was found as BA and 11 with non-BA. Here 30 control subjects were taken. In the 40 patients with neonatal jaundice, we did not find any significant differences regarding age and total bilirubin level, but male to female ratio were significantly differ between patients with BA and patients with non-BA. Regarding compare with control subjects with BA patients, though male to female ratio was not significantly differ but age and total bilirubin level had significant difference between the groups (Table 1).

Nineteen patients with BA had positive TC sign on US, neither the patients with non-BA or control subjects had TC sign. 
Table 1. Clinical profile of patients with BA, patients with non-BA and control subjects at US.

\begin{tabular}{|c|c|c|c|c|c|}
\hline Variables & Patients with BA (29) & Patients with Non-BA (11) & p value ${ }^{\alpha}$ & Control Group (30) & p value ${ }^{\beta}$ \\
\hline Age & $42.55 \pm 8.44$ & $34.00 \pm 6.88$ & 0.316 & $37.10 \pm 9.94$ & 0.001 \\
\hline Male to female ratio & $16: 13$ & 09:02 & 0.001 & 19:11 & 0.252 \\
\hline Total bilirubin & $7.57 \pm 2.62$ & $6.24 \pm 2.85$ & 0.612 & 0.34 & 0.001 \\
\hline
\end{tabular}

Table 2. Compare of US and color Doppler findings of patients with BA, patients with non-BA and with control subjects.

\begin{tabular}{|c|c|c|c|c|c|c|}
\hline Variables & Patients with BA (29) & Patients with Non-BA (11) & p value ${ }^{\alpha}$ & Control Group (30) & p value ${ }^{\beta}$ & p value ${ }^{\Sigma}$ \\
\hline \multicolumn{7}{|c|}{ TC sign } \\
\hline Present & $19(65.5)$ & $3(27.3)$ & NA & $0(00)$ & NA & NA \\
\hline Absent & $10(34.5)$ & $8(72.7)$ & NA & $30(100)$ & NA & NA \\
\hline \multicolumn{7}{|c|}{ GB length } \\
\hline$<1.5 \mathrm{~cm}$ & $20(69.0)$ & $4(36.4)$ & \multirow{2}{*}{0.564} & $00(00)$ & \multirow{2}{*}{0.001} & \multirow{2}{*}{0.001} \\
\hline$\geq 1.5 \mathrm{~cm}$ & $9(31)$ & $7(63.6)$ & & $30(100)$ & & \\
\hline \multicolumn{7}{|c|}{ Diameter of Portal vein $\left(\mathbf{m m}^{2}\right)$} \\
\hline Absent & $4.48 \pm 0.79$ & $3.91 \pm 1.23$ & 0.113 & $3.92 \pm 0.49$ & 0.005 & 0.001 \\
\hline \multicolumn{7}{|c|}{ Diameter of hepatic artery $\left(\mathrm{mm}^{2}\right)$} \\
\hline Present & $2.22 \pm 0.43$ & $1.97 \pm 0.44$ & 0.0857 & $1.51 \pm 0.30$ & 0.0029 & 0.13 \\
\hline \multicolumn{7}{|c|}{ Hepatic subscapular flow } \\
\hline Present & $29(100)$ & $0(00)$ & NA & $0(00)$ & NA & NA \\
\hline Absent & $0(00)$ & $11(100)$ & NA & $30(100)$ & NA & NA \\
\hline $\begin{array}{l}\alpha, p: \text { Values for } c c \\
\beta, p: \text { Values for } c c \\
\sum p: \text { Values for co }\end{array}$ & $\begin{array}{l}\text { patients who had BA w } \\
\text { control subjects with pa } \\
\text { control subjects with pat }\end{array}$ & $\begin{array}{l}\text { ents who had non-BA } \\
\text { who had BA } \\
\text { who had non- BA }\end{array}$ & & & & \\
\hline
\end{tabular}

Table 3. Multiple logistic regression showing association of predictors with presence of BA or not.

\begin{tabular}{|c|c|c|}
\hline Variables & Wald $/ x^{2}$ value & Odds Ratio (OR) \\
\hline \multicolumn{3}{|c|}{ Sex } \\
\hline \multicolumn{3}{|c|}{ Male (reference) } \\
\hline Female & 0.001 & 1.113 \\
\hline \multicolumn{3}{|c|}{ TC sign } \\
\hline \multicolumn{3}{|c|}{ Present (reference) } \\
\hline Absent & 0.001 & 2.906 \\
\hline \multicolumn{3}{|c|}{ GB length } \\
\hline \multicolumn{3}{|c|}{$<1.5 \mathrm{~cm}$ (reference) } \\
\hline$\geq 1.5 \mathrm{~cm}$ & 0.001 & 3.274 \\
\hline \multicolumn{3}{|c|}{ Hepatic subscapular flow } \\
\hline \multicolumn{3}{|c|}{ Present (reference) } \\
\hline Absent & 0.001 & 8.851 \\
\hline
\end{tabular}

The GB length was significantly shorter in control subjects $(\mathrm{p}<0.001)$. There was no significant difference in mean diameter of portal vein in patients with BA and patients with non-BA but control subjects had with both groups (Table 2).

On multiple logistic regression it was noted that gender, presence of TC sign, hepatic subscapular flow presence or absent and GB length were significant predictors for developing BA (Table 3). Patients who had positive TC sign on US had 2.906 times more chance to develop BA than who had not TC signs. On color Doppler scan that had positive hepatic subscapular flow had 8.87 times more chance to develop BA (Table 3 ).

\section{Discussion}

In this study, mean age of infants of BA and non-BA is significantly different in both groups $(p<0.001)$ and male to female ratio is significantly indifferent in both groups. Presence of the TC sign is a strong predictor for diagnosis of BA [4]. In the current study, $65.5 \%$ of BA neonates present with TC sign (Figure 3).

There are several reports regarding the arterial changes in 50 patients with BA $[7,8]$ the diameter of the hepatic artery is significantly larger in patients with BA than in patients with non-BA and control subjects [9]. In this study we have noted that man diameter of hepatic artery in BA is $2.22 \pm 0.43$ and in non-BA is $1.97 \pm 0.44$ and in control $1.51 \pm 0.30 \mathrm{~mm}$. Our results also showed that the diameter of portal vein was significantly differ in BA and non-BA groups $\mathrm{p}=0.005$ ).

Ulflacker and Pariente [10] described that presence angiographically demonstrable perivascular arterial tufts in the periphery of the hepatic arterial circulation in patients with BA and suggested that these findings might be useful in the diagnosis of BA. In the current study, color Doppler US was used to see the enlargement of hepatic artery and hepatic arterial flow that extended to the hepatic surface were seen in all patients with BA.

All patients with BA who had hepatic subscapular flow on color Doppler US images had subscapular telangiectatic vessels at the time of the Kasai procedure. The pathogenesis of hepatic arteriopathy in patients with BA is unknown. In the current study in multiple logistic regressions it is found that OR for 
TC sign is 2.906 where for subscapular flow same parameter is 8.851 . So it can be said that presence of subscapular flow is more potent predictor than TC sign for diagnosis of BA.

There was no significant difference between patients with non-BA and BA in terms of diameter of portal vein $(\mathrm{p}=0.113)$, diameter of hepatic artery $(p=0.0857)$ GB length $(p=0.564)$.

\section{Conclusion}

Previously TC sign was used as a determinant of diagnosis of BA. But now days, hepatic arterial flow extending to the hepatic surface on color Doppler is a good tool for diagnosis of BA. So the detection of BA can be supplemented by performing color Doppler US in addition to routine US when evaluating hepatic subscapular flow; by using color Doppler US we can potentially prevent delayed diagnosis of BA.

\section{References}

1. Emerick KM, Whitington PF. Neonatal liver disease. Pediatr Ann. 2006;35(4):280-6.

2. Kim MJ, Park YN, Han SJ, et al. Biliary atresia in neonates and infants: Triangular area of high signal intensity in the porta hepatis at T2-weighted MR cholangiography with US and histopathologic correlation. Radiology. 2000;215(2):395-401.

3. Moyer V, Freese DK, Whitington PF, et al. Guideline for the evaluation of cholestatic jaundice in infants:
Recommendations of the North American Society for Pediatric Gastroenterology, Hepatology and Nutrition. J Pediatr Gastroenterol Nutr. 2004;39(2):115-28.

4. Park WH, Choi SO, Lee HJ. The ultrasonographic 'triangular cord' coupled with gallbladder images in the diagnostic prediction of biliary atresia from infantile intrahepatic cholestasis. J Pediatr Surg. 1999;34(11):1706-10.

5. Tang ST, Ruan QL, Cao ZQ, et al. Diagnosis and treatment of biliary atresia: A retrospective study. Hepatobiliary Pancreat Dis Int. 2005;4(1):108-12.

6. Kim WS, Cheon JE, Youn BJ, et al. Hepatic arterial diameter measured with US: Adjunct for US diagnosis of biliary atresia. Radiology. 2007;245(2):549-55.

7. Humphrey TM, Stringer MD. Biliary atresia: US diagnosis. Radiology. 2007;244(3):845-51.

8. Ho CW, Shioda K, Shirasaki K, et al. The pathogenesis of biliary atresia: A morphological study of the hepatobiliary system and the hepatic artery. J Pediatr Gastroenterol Nutr. 1993;16(1):53-60.

9. Santos JL, Silveira TR, Silva VD, et al. Medial thickening of hepatic artery branches in biliary atresia: A morphometric study. J Pediatr Surg. 2005;40(4):637-42.

10. Uflacker R, Pariente DM. Angiographic findings in biliary atresia. Cardiovascular and Interventional Radiology. 2004;27(5):486-90.

\section{*Correspondence to:}

Mamuda Monowara

Department of Radiology and Imaging,

Bangladesh Institute of Child Health,

Shishu Hospital,

Dhaka, Bangladesh

Tel: +8801676097932

E-mail: mahmudamonowara085@gmail.com 